DOI: https://doi.org/10.11144/Javeriana.umed58-3.dfhr

\title{
Deterioro funcional hospitalario. Revisión y actualización con una perspectiva orientada a mejorar la calidad de atención del anciano
}

Hospital Functional Impairment. Review and Update with a Perspective Aimed at Improving Quality of Care of the Elderly

Fecha de recepción: 16 Noviembre 2016 | Fecha de aprobación: 15 Febrero 2017

\author{
María Juliana Lozano Rengifo a \\ Hospital Universitario San Ignacio, Colombia \\ Diego Andrés Chavarro-Carvajal \\ Hospital Universitario San Ignacio, Colombia
}

a Autora de correspondencia. Correo electrónico:
mjlozanorengifo@gmail.com

Cómo citar: Lozano Rengifo MJ, Chavarro-Carvajal DA. Deterioro funcional hospitalario. Revisión y actualización con una perspectiva orientada a mejorar la calidad de atención del anciano. Univ Med. 2017;58(3):1-6. doi: https://doi.org/10.11144/Javer iana.umed58-3.dfhr

\section{RESUMEN}

El deterioro funcional hospitalario se caracteriza por la pérdida de, al menos, una de las actividades básicas de la vida diaria con respecto a la funcionalidad previa al inicio de la enfermedad aguda. Tiene factores de riesgo asociados que son modificables durante la hospitalización y de ahí la importancia de la identificación temprana y la intervención oportuna. Por eso se debe conocer esta entidad clínica, debido a las complicaciones asociadas que se pueden presentar tanto a corto como a largo plazo, entre las cuales están aumento de la estancia hospitalaria, presencia de complicaciones médicas, mayor riesgo de institucionalización, mayores costos para el sistema de salud y aumento de la mortalidad. El objetivo de esta revisión es conocer la forma de presentación, los factores de riesgo, la identificación temprana y los programas de intervención para mejorar la calidad de atención en los pacientes ancianos hospitalizados por enfermedad médica.

Palabras clave

dependencia; hospitalización; anciano; patología clínica.

\begin{abstract}
Functional decline in hospitalized elderly adults is characterized by the loss of at least one of the basic activities regarding the functionality two weeks prior to the onset of acute illness. It has associated risk factors that could be modified during hospitalization, therefore the importance of the early identification and well-timed intervention. It is important to know the clinical entity's background, due to the short or long term multiple associated complications that could unexpectedly appear; for example, increased hospital stay, medical complication presence, increased institutionalization risk, higher costs for the health system and increased mortality rate. The objective of this work is to know about the presence, risk factors, early identification and the intervention programs to improve the caring quality in elderly patients hospitalized for medical illness.

Keywords

dependency; hospitalization; eldery; clinical pathology.
\end{abstract}




\section{Introducción}

Los cambios demográficos en las últimas décadas, tanto en Colombia como en el mundo, han estado influenciados por al aumento de la expectativa de vida. En nuestro país, desde los años treinta, empezó el descenso de la mortalidad, y a partir de los años sesenta, una disminución de la fecundidad, lo que ha producido cambios en la pirámide poblacional con un aumento significativo del número de personas mayores de 60 años [1].

Con estos cambios, se ha visto un aumento de la prevalencia de las enfermedades crónicas, deterioro funcional, deterioro cognitivo, polifarmacia y disminución de la reserva funcional; lo que puede llevar a mayor dependencia y disminución de la calidad de vida en los pacientes ancianos.

Se estima que aproximadamente el $10 \%$ de los pacientes mayores de 60 años requieren, al menos, una hospitalización al año por un proceso agudo [2]. Dentro de las complicaciones hospitalarias más frecuentes encontramos el deterioro funcional hospitalario (DFH), con una prevalencia de entre el $35 \%$ y el $70 \%$ [2].

Este proceso se define como la pérdida de la capacidad de realización de, al menos, una de las actividades básicas de la vida diaria con respecto a la situación basal (dos semanas antes del inicio de la enfermedad aguda). Se habla de dos etapas en el deterioro funcional: la primera, que ocurre antes de la hospitalización, que se presenta desde el inicio de la enfermedad aguda hasta el ingreso al hospital, y la segunda, que corresponde al deterioro que se produce durante la hospitalización [3]. Esta diferenciación es importante, debido a que la primera es no modificable; mientras que segunda se puede modificar con la identificación temprana del paciente en riesgo y las intervenciones oportunas.

Se debe tener en cuenta que las complicaciones del deterioro funcional hospitalario pueden superar la enfermedad aguda. Dentro de las más frecuentes encontramos peor evolución de la enfermedad, mayor estancia hospitalaria, mayor consumo de recursos, mayores tasas de reingreso, mayor dependencia funcional, mayor riesgo de institucionalización y aumento de la mortalidad [4].

\section{Epidemiología}

El DFH tiene una prevalencia aproximada del $35 \%$ al $70 \%$ de los pacientes mayores de 60 años hospitalizados por enfermedad médica [2], con una incidencia al ingreso hospitalario del $35 \%$ al $50 \%$. Este deterioro se encuentra más en relación con las características del individuo y la hospitalización, que con la gravedad de la enfermedad aguda, por lo cual los estudios no la consideran un factor de riesgo independiente para desarrollar deterioro funcional hospitalario [5].

Se estima que, al egreso, los mayores de 70 años presentan deterioro en alguna de las actividades básicas de la vida diaria, en un $46 \%$; mientras que en mayores de 90 años esta cifra asciende hasta un $88 \%$. Al mes de egresados, el $49 \%$ de los pacientes persiste con deterioro en alguna de las actividades básicas; mientras que en actividades instrumentales se evidencia persistencia de deterioro, en un $57 \%$.

Se ha hecho seguimiento a los 3 meses y al año, y se ha encontrado a los 3 meses que el $19 \%$ persiste con deterioro en alguna de las actividades básicas; mientras que un $40 \%$ presenta deterioro en las instrumentales, y al año se ha visto que hasta un $28 \%$ persiste con dificultades para la realización de alguna de las actividades básicas de la vida diaria [4].

En cuanto a la mortalidad, las cifras varían según los estudios, debido a los diferentes métodos de evaluación y análisis; sin embargo, en un estudio publicado en 1996 con seguimiento a 3 meses se evidenció un aumento del riesgo de mortalidad en un $3 \%$ en los pacientes que sufrieron deterioro funcional global, frente a los que no [6]. Otros estudios con seguimiento a un año han reportado un incremento de la mortalidad en un $20 \%$ en los pacientes con DFH [7]; mientras que otros evidenciaron un aumento de la mortalidad al año, entre el $17 \%$ y el $54 \%$ [8]. 
En cuanto al riesgo de institucionalización, se ha visto que, posterior al egreso, los pacientes con DFH presentan un $14 \%$ más de probabilidad de ser institucionalizados [9], y mayor riesgo de nueva hospitalización, en un $20 \%$ al $27 \%$ [6].

\section{Factores de riesgo}

Existen muchos factores de riesgo que predisponen al individuo a sufrir DFH. Unos dependen del individuo y los cambios relacionados con la edad y los otros se relacionan con la hospitalización en sí misma.

Dentro de los factores asociados con la edad, encontramos los cambios fisiológicos que la acompañan, como son disminución de la masa ósea, de la fuerza muscular, de la densidad mineral ósea, de la capacidad aeróbica, de la sensibilidad barorreceptora, del agua corporal, de la ventilación pulmonar; presencia de inestabilidad vasomotora, y alteraciones neurosensoriales [4].

Entre los factores asociados a la hospitalización, encontramos como principal factor de riesgo la inmovilidad, definida esta como la permanencia en cama todo el día o levantarse solo una vez en el día al sillón. Se ha encontrado una relación directa entre el tiempo en cama y la disminución del Barthel respecto al ingreso, y aunque no hay muchas referencias en la literatura sobre la cuantificación de inmovilismo durante la hospitalización, algunos estudios han demostrado que hasta el $83 \%$ del tiempo pasan los pacientes en la cama, a pesar de tener capacidad para deambular [10]. La inmovilidad es un factor de riesgo independiente del DFH y su importancia, debida a los cambios que produce en la capacidad aeróbica y en la reserva muscular, principalmente de los miembros inferiores dentro de las primeras 48 horas de reposo en cama.

Otro factor importante, debido a que es independiente y tiene valor pronóstico, es el deterioro funcional previo o situación basal que, cómo se dijo, es la funcionalidad dos semanas previas al ingreso. En este punto se ha documentado que los pacientes con una buena situación previa son 2,5 veces más susceptibles de recuperar su situación basal al alta; mientras que los pacientes que tienen un Barthel previo menor de 60 tienen mayor riesgo de deteriorarse durante la hospitalización y persistir con deterioro al egreso [11]. En este punto vale considerar el efecto suelo, el cual hace referencia a los pacientes que ingresan con una dependencia funcional muy severa y, por tanto, no pueden tener mayor deterioro durante la hospitalización. Es importante diferenciar entre el deterioro funcional previo y el deterioro funcional al ingreso; este último se considera el estado que presenta el paciente en el momento del ingreso a la hospitalización. Un índice de Barthel menor a 65 al ingreso es un factor independiente de deterioro funcional y mayor riesgo de discapacidad y muerte al alta y al mes [4].

Dentro de la hospitalización es muy frecuente encontrar otros factores asociados, como las rutinas hospitalarias, que son todas aquellas indicaciones que limitan la movilidad de los pacientes, además de la polifarmacia y el riesgo de delírium, que se han relacionado con mayores complicaciones y aumento de la estancia hospitalaria [12].

\section{Identificación del paciente en riesgo}

Debido a la rápida instauración de las complicaciones asociadas al inmovilismo, se ha considerado una prioridad la identificación temprana de los factores de riesgo, la cual debe realizarse dentro de las primeras 48 horas de ingreso. El objetivo principal es discriminar a aquellos que se benefician más de las intervenciones tempranas, con lo cual se busca disminuir las complicaciones asociadas, la estancia hospitalaria, los costos del sistema sanitario y la mortalidad. Para esto, existen muchas herramientas de tamizaje que son fáciles y rápidas de implementar, que han demostrado ser más efectivas que tener en cuenta los factores de riesgo de forma independiente; sin embargo, es importante resaltar que aún no se encuentran validadas en nuestro medio [13]. A continuación, se exponen algunas de ellas. 
La primera es la herramienta Hospital Admission Risk Profile (HARP), validada en 1996 para estratificar a los ancianos en el momento del ingreso al hospital según el riesgo de desarrollar alguna discapacidad al egreso y a los tres meses. Esta escala consta de tres puntos principales, que son la edad, el puntaje del Mini-Mental State Examination (MMSE) abreviado y el deterioro de las actividades instrumentales al ingreso. Cada ítem tiene un puntaje que, al sumarlos, clasifica al paciente en alto riesgo, riesgo intermedio y bajo riesgo. El objetivo principal es identificar a los pacientes en riesgo de deterioro funcional para inicio de intervención temprana [14].

También se aplica la herramienta Identification of Seniors at Risk or Functional Decline (SHERPA), que cuenta con cinco ítems: edad, dependencia previa en las actividades instrumentales de la vida diaria (AIVD), caídas en el último año, deterioro cognitivo con MMSE abreviado y autopercepción del estado de salud. Clasifica al paciente en bajo riesgo, leve, moderado y alto.

Encontramos también algunas herramientas de tamizaje que están diseñadas para una población específica del servicio de urgencias; entre estas tenemos la Identification of Seniors at Risk (ISAR), que fue validada en Canadá en 1999 para identificar el riesgo de complicaciones, como institucionalización, deterioro funcional y mortalidad, en las personas ancianas tras los 6 meses de la visita al servicio de urgencias. Es una herramienta que puede ser de autorreporte o aplicada por el personal de salud y tiene 6 ítems (cada uno de los cuales da un punto: edad, dependencia funcional previa y actual, ingreso reciente, problema de memoria, problemas de visión, polifarmacia), y el punto de corte es más de dos [15].

La otra herramienta que cabe mencionar es la Triage Risk Screening Tool (TRST), la cual tiene como población índice los pacientes que consultan a urgencias y tienen egreso. Esta cuenta también con seis ítems (deterioro cognitivo, dificultad para caminar, caídas recientes, polifarmacia, visitas recientes a urgencias y condición de riesgo según enfermería) y busca identificar el riesgo de deterioro funcional tras la visita a urgencias.

Existen muchas otras herramientas que pueden ser útiles para identificar riesgo de DFH y sus complicaciones. Las expuestas anteriormente son de fácil aplicación y hasta el momento han sido las más usadas; sin embargo, como se dijo aún faltan estudios para validación en nuestro medio.

\section{Intervenciones}

Una vez identificado el paciente en riesgo, el siguiente paso es iniciar la intervención de forma temprana. Recordemos que los cambios asociados al inmovilismo comienzan en las primeras 48 horas, por lo cual la identificación del paciente debe ser al ingreso de la hospitalización y la intervención debe iniciarse en los primeros dos días.

\section{Programa de ejercicios}

El programa de ejercicio es un entrenamiento físico que busca fortalecer los miembros inferiores, ya que de estos depende en gran parte la funcionalidad y disminuye el riesgo de caídas. Es una medida muy eficaz que ha demostrado prevenir y disminuir el deterioro funcional [16] por medio del aumento de la masa, la potencia y la fuerza muscular. Se recomienda que los ejercicios sean progresivos, según la tolerancia del paciente, para evitar eventos adversos.

No existe un consenso claro sobre la rutina de ejercicios que se debe emplear; sin embargo, diferentes estudios han planteado rutinas que, comparadas con inmovilismo, han demostrado traer más beneficios. Nuevos modelos incluyen el ejercicio como parte del manejo convencional de los adultos mayores hospitalizados por enfermedad médica aguda, el cual debe realizarse al menos hasta que el paciente tenga egreso hospitalario [17]. Los estudios concluyen que el ejercicio debe ser individualizado y supervisado por un fisioterapeuta.

Es importante tener en cuenta las contraindicaciones para inicio del ejercicio, entre 
las que encontramos infarto agudo de miocardio reciente o angina inestable, hipertensión no contralada, insuficiencia cardiaca aguda, bloqueo auriculoventricular completo, signos de respuesta inflamatoria sistémica, estado neurológico comprometido, tromboembolismo pulmonar, trombosis venosa profunda, fracturas y cirugías.

\section{Terapia ocupacional}

La terapia ocupacional tiene como objetivo lograr la máxima independencia posible en las actividades básicas de la vida diaria hasta las instrumentales; también busca mejorar la participación social, el trabajo y las actividades en el tiempo libre. Lo anterior con el fin mejorar la calidad de vida del paciente y su entorno.

Esta terapia se realiza en la habitación y el baño del paciente con sus utensilios de cuidado personal. Se recomiendan sesiones diarias de 30 a 40 minutos cada una y se debe realizar de forma individual [18].

\section{Nutricional}

El aspecto nutricional es muy importante en el anciano. Debemos recordar que la ingesta de proteínas es esencial para mantener y fortalecer el músculo. Se recomienda que el anciano consuma, en promedio, $1,2 \mathrm{~g} / \mathrm{kg} / \mathrm{día}$, y las personas mayores de 65 años, en las cuales se busca mantener y recuperar la masa magra con ejercicio aeróbico, el consumo de proteínas debe ser mayor a $1,2 \mathrm{~g} / \mathrm{kg} /$ día [19].

\section{Manejo multidisciplinario}

En estudios recientes se ha indicado que el relieve está puesto en el manejo multidisciplinario para prevenir el DFH. Las unidades geriátricas de agudos han demostrado disminuir en un $12 \%$ el deterioro funcional con disminución de la mortalidad y los costos en salud, comparado con los pacientes hospitalizados por medicina interna [20].

\section{Conclusiones}

El DFH es una entidad clínica muy frecuente que tiene múltiples complicaciones asociadas, por lo cual en las últimas décadas ha cobrado gran importancia. A pesar de la evidencia, en nuestro medio aún falta crear conciencia en el personal de salud sobre la identificación de los pacientes en riesgo, los factores modificables y la intervención temprana.

Mediante la educación y socialización de este tema, se busca mejorar el pronóstico de nuestros pacientes, la calidad de vida y la calidad de la atención en salud. Sin embargo, aún faltan estudios en nuestro país sobre las tasas de complicaciones asociadas al DFH y sobre las intervenciones más eficaces en nuestro medio.

\section{Referencias}

1. Gaviria Uribe A. Envejecimiento demográfico: Colombia 1951-2020 dinámica demográfica y estructuras poblacionales. Bogotá: Ministerio de Salud y Protección Social; 2013.

2. Córcoles-Jiménez MP. Deterioro funcional asociado a la hospitalización enpacientes mayores de 65 años. Enferm Clin. 2015;26(2):121-8.

3. Mauricio Ocampo J. Revisión sistemática de literatura: declinación funcional en ancianos hospitalizados. Revista médica Risaralda. 2016;22(1):49-57. DOI: http://d x.doi.org/10.22517/25395203.13681

4. Osuna-Pozo CM, Ortiz-Alonso J, Vidán M, Ferreira G, Serra-Rexach A. Revisión sobre el deterioro funcional en el anciano asociado al ingreso porenfermedad aguda. Rev Esp Geriatr Gerontol. 2014;49(2):77-89.

5. Vidán Astiz MT, Sánchéz García E, Alonso Armesto M, Montero Errasquin B, Martínez de la Casa A, Ortiz FJ, et al. Deterioro funcional durante la hospitalización en ancianos. Beneficios del ingreso en el servicio de geriatría. Rev Esp Geriatr Gerontol. 2008;43:133-8. 
6. Sager MA, Franke T, Inouye SK, Landefeld CS, Morgan TM, Rudberg MA, et al. Functional outcomes of acute medical illness and hospitalization in older persons. Arch Intern Med. 1996;156(6):645-52.

7. Boyd CM. Recovery in activities of daily living among older adults following hospitalization for acute medical illness. J Am Geriatr Soc. 2008:2171-9.

8. Covinsky KE, Justice AC, Rosenthal GE, Palmer RM, Landefeld CS. Measuring prognosis and case mix in hospitalized elders: The important of funtional status. J Gen Intern Med. 1997;12(4):203-8.

9. Gómez JF. Salud del anciano: valoración. Manizales, Caldas: Blanecolor; 2014.

10. Martínez-Velilla N, Urbiston-Lasa G, Veitemilla-Erice E, Cambra-Contín K. Cuantificación de las horas de encamamiento en pacientes hospitalizados por afección médica y deterioro funcional y mortalidad secundarios. Rev Esp Geriatr Gerontol. 2013;48(2):96.

11. Baztán JJ, González M, Morales C, Vázquez E, Morón N, Forcano S, Ruipérez J. Variables asociadas a la recuperación funcional y la institucionalización al alta en ancianos ingresados en una unidad geriátrica de media estancia. Rev Clin Esp. 2004;204(11):574-82.

12. Abizanda Soler P. La pérdida funcional al ingreso, principal variable explicativa de discapacidad y mortalidad al alta y al mes en ancianos hospitalizados. Rev Esp Geriatr Gerontol. 2007;201-11.

13. Martín-Sánchez FJ, Fernández Alonso C, Gil Gregorio P. Puntos clave en la asistencia al anciano frágil en Urgencias. Med Clin (Barc). 2013;140(1):24-9.

14. Sager MA, Rudberg MA, Jalaluddin M, Franke T, Inouye SK, Landefeld CS, et al. Hospital Admission Risk Profile (HARP): Identifying older patients at risk for functional decline following acute medical illness and hospitalization. J Am Geriatr Soc. 1996;44(3):251-7.

15. Yao JL, Fang J, Lou QQ, Anderson RM. A systematic review of the identification of seniors at risk (ISAR) tool for the prediction of adverse outcome in elderly patients seen in the emergency department. Int J Clin Exp Med. 2015;8(4):4778-86.

16. McCullagh R, O'Connell E, O'Meara S, Perry I, Fitzgerald A, O'Connor K. A study protocol of a randomised controlled trial to measure the effects of an augmented prescribed exercise programme (APEP) for frail older medical patients in the acute setting. BMC Geriatr. 2016;16:79.

17. Martínez-Velilla N, Cadore L, CasasHerrero Á, Idoate-Saralegui F, Izquierdo M. Physical activity and early rehabilitation in hospitalized elderly medical patients: systematic review of randomized clinical trials. J Nutr Health Aging. 2016;20(7):738-51.

18. Domínguez Martín L, Lozano Berrío V. El anciano que precisa terapia ocupacional. En: Abizanda Soler P, Rodríguez Mañas L, editores. Tratado de medicina geriátrica: fundamentos de la atención sanitaria a los mayores. Madrid: Elsevier; 2014. p. 277-85.

19. León Ortiz M, Ariza Zafra M. Valoración nutricional en el anciano. En: Abizanda Soler P, Rodríguez Mañas L, editores. Tratado de medicina geriátrica: fundamentos de la atención sanitaria a los mayores. Madrid: Elsevier; 2014. p. 204-12.

20. Romero Rizos L, Sánchez Jurado PM, Abizanda Soler P. El anciano en la unidad de geriatría de agudos. Rev Esp Geriatr Gerontol. 2009;44 Supl 1:15-26. 